\title{
Erratum to: TTG1-mediated flavonols biosynthesis alleviates root growth inhibition in response to $\mathrm{ABA}$
}

\author{
Hoai Nguyen Nguyen · Jun Hyeok Kim • \\ Woo Young Hyun · Ngoc Trinh Nguyen \\ Suk-Whan Hong $\cdot$ Hojoung Lee
}

Published online: 14 February 2013

(C) Springer-Verlag Berlin Heidelberg 2013

\section{Erratum to: Plant Cell Rep}

\section{DOI 10.1007/s00299-012-1382-1}

Figure $6 \mathrm{c}$ is missing in the original publication. The complete figure is reproduced on the following page.

The online version of the original article can be found under doi:10.1007/s00299-012-1382-1.

H. N. Nguyen · J. H. Kim · W. Y. Hyun · H. Lee $(\bowtie)$

College of Life Sciences and Biotechnology, Korea University,

1,5-Ka Anam-Dong, Seongbuk-Gu,

Seoul 136-713, Republic of Korea

e-mail: lhojoung@korea.ac.kr

N. T. Nguyen $\cdot$ S.-W. Hong $(\bowtie)$

Department of Molecular Biotechnology, College of Agriculture

and Life Sciences, Bioenergy Research Center, Chonnam

National University, Gwangju, Republic of Korea

e-mail:sukwhan@chonnam.ac.kr 
Fig. 6 Growth phenotypes of wild-type, TTG1-OX, PAP1$D$ and $t$ tg 1 seedlings grown on $1 / 2 \times$ MS medium

supplemented with $10 \mu \mathrm{M}$ ABA or $300 \mathrm{mM}$ mannitol. a Fourday-old seedlings were transferred to test medium and allowed to grow for 10 days before being photographed. The seedlings shown were typical of three separate experiments performed at $23{ }^{\circ} \mathrm{C}(n=15$ seedlings per experiment). b The numbers of LRs in these seedlings were counted under a microscope. The data shown are from three separate experiments performed at $23{ }^{\circ} \mathrm{C}(n=10$ per experiment). One-way ANOVA Tukey's test $(P<0.05)$ was performed to indicate a significant difference in LR number between wild-type and mutants per treatment. Lowercase letters $(a, b, c)$ were marked above the column to reflect significant differences. Vertical bars indicate standard error $(n=30)$. $\mathbf{c}$ The transcript levels of the stress-marker genes Cor15A and KIN1 were determined for wild-type and TTG1-OX \#4 and \#5 seedlings on normal $1 / 2 \times$ MS medium. Several rounds of $R T-P C R$ were performed, and $P C R$ results from two experiments using different numbers of cycles are shown. UBC21 was used as the internal loading control. Tenday-old seedlings were grown on normal $1 / 2 \times$ MS medium before total RNA extraction for $R T-P C R$

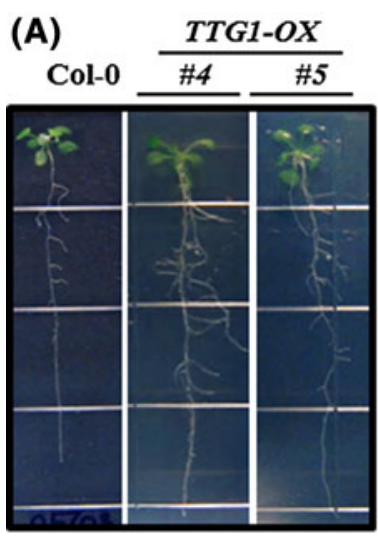

Control

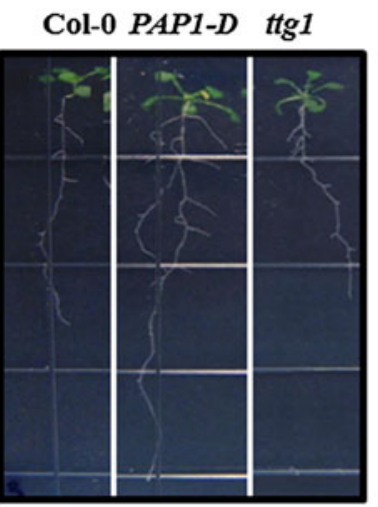

Control

(B)

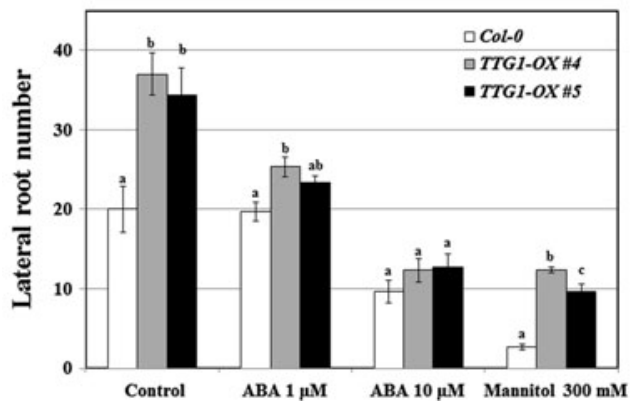

(C)

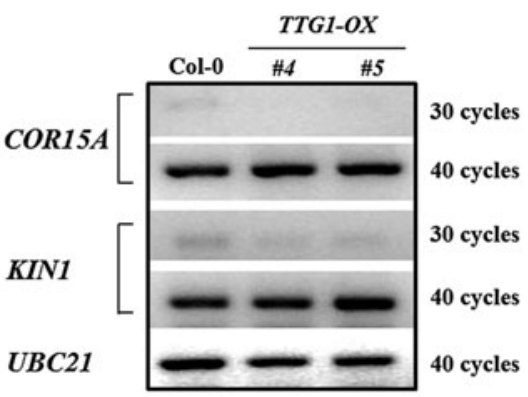

